\title{
BMJ Open Treatment patterns and medication adherence among newly diagnosed patients with migraine: a drug utilisation study
}

\author{
Valentina Orlando, ${ }^{1,2}$ Sara Mucherino (D) , ${ }^{1,2}$ Valeria Marina Monetti, ${ }^{1,2}$ Ugo Trama, ${ }^{3}$ \\ Enrica Menditto ${ }^{1,2}$
}

To cite: Orlando V,

Mucherino S, Monetti VM, et al. Treatment patterns and medication adherence among newly diagnosed patients with migraine: a drug utilisation study. BMJ Open 2020;10:e038972. doi:10.1136/ bmjopen-2020-038972

- Prepublication history and additional material for this paper is available online. To view these files, please visit the journal online (http://dx.doi.org/10. 1136/bmjopen-2020-038972)

Received 30 March 2020 Revised 30 June 2020 Accepted 17 October 2020
Check for updates

(c) Author(s) (or their employer(s)) 2020. Re-use permitted under CC BY-NC. No commercial re-use. See rights and permissions. Published by BMJ.

\section{${ }^{1}$ CIRFF, Center of}

Pharmacoeconomics, University of Naples Federico II, Naples, Italy

${ }^{2}$ Department of Pharmacy, University of Naples Federico II, Naples, Italy

${ }^{3}$ Regional Pharmaceutical Unit, Campania Region, Naples, Italy

Correspondence to

Dr Sara Mucherino;

sara.mucherino@unina.it

\section{ABSTRACT}

Objectives Prophylactic drugs currently used for migraine treatment are not specific. Furthermore, few studies in existing literature describe drugs utilisation patterns and adherence to migraine prophylactic treatment. This study is aimed to describe utilisation patterns of migraine drugs, evaluate adherence to prophylactic medications and investigate drug-related costs.

Design Retrospective population-based study using an administrative health-related database.

Setting Primary care setting in the Campania region, Southern Italy.

Participants This study was carried out between 1 January 2016 and 31 December 2018, involving 12894 subjects with any primary or secondary hospital discharge with migraine diagnosis, or at least two medical dispensations of migraine-specific acute or prophylactic medications (triptans or pizotifen). Subjects were classified into four treatment cohorts: no treatment, acute, prophylactic and both acute and prophylactic. Subjects were followed-up for 1 year.

Outcome measures Utilisation patterns of migraine drugs at treatment initiation; adherence to prophylactic treatment; discontinuation, restart and switching rates; annual migraine drug costs per patient.

Results Overall, $81.1 \%$ of subjects received acute treatment as their initial migraine treatment regimen, $10.7 \%$ prophylactic treatment, $8.2 \%$ both acute and prophylactic treatment. 599 patients were treated prophylactically; of these, $26.2 \%$ adhered to their initial treatment while $73.8 \%$ reported interruptions in treatment. Among the latter, $46.4 \%$ of patients discontinued the treatment completely within 103 days (IQR 89.0 ), 31\% restarted treatment 46 days after interruption (IQR 60.0) and 22.6\% switched to another treatment within 98 days (IQR 57.5) $(p<0.001)$. The median annual cost of drugs per patient was $€ 103$ for those treated acutely, $€ 75$ for those treated prophylactically, $€ 163$ for those treated both.

Conclusions Migraine treatment with acute medications is still prevalent in Italy; only few patients received prophylactic treatment with poor adherence to treatment. These findings reflect an unmet need for improved prophylactic therapies in order to provide a better disease management.
Strengths and limitations of this study

- The study describes the treatment of migraine in the Italian clinical practice by evaluating medical data from a stable and specific geographical population.

- By characterising the use of treatments for migraine in this context, the study provides useful data for evaluating the dynamics of migraine treatment in a real-life setting.

- Data source used in this study does not track diagnostic information. Consequently, patient identification had to be performed ex post by the use of proxy drugs that were reimbursed by the National Health Service (NHS)

- The strategy used to identify patients with migraine may have omitted those who used drugs that were not reimbursable by the NHS.

- The final dataset may have lacked information relating to the reasons for discontinuation of treatment.

\section{INTRODUCTION}

Migraine is a common disabling primary headache disorder. Numerous studies have documented the high prevalence and socioeconomic and personal impacts of migraine, resulting in an estimated economic impact of $€ 27$ billion per year in the European Union alone. ${ }^{1-5}$ According to the WHO's ranking of the main causes of disability, headache disorders are among the 10 most disabling conditions. ${ }^{6}$ According to the Italian Guidelines for Primary Headaches, ${ }^{7}$ a range of treatments are available for migraine. Furthermore, the guidelines for the pharmacological treatment of migraine describe both acute and prophylactic approaches. ${ }^{7}$ Although prophylactic therapy is primarily used to reduce the frequency, duration or severity of attacks, it also enhances a patient's response to acute treatments while improving socioeconomic function. ${ }^{8}$ However, prophylactic drugs are not migraine-specific, because they are mainly indicated for other conditions such as 
depression, epilepsy and hypertension. There is evidence that these medications are frequently associated with side effects and low adherence to therapy, thus leading to poor efficacy. ${ }^{9}$ In recent years, a range of novel therapies have emerged for the specific treatment of migraine by both acute and prophylactic regimens. ${ }^{10}$ For example, the injection of botulinum toxin A was approved in 2013 as a preventive therapy for migraine. ${ }^{11}$ More recently, monoclonal antibodies directed against the calcitonin generelated peptide (CGRP) have been introduced as a novel therapeutic strategy for migraine, thus indicating potential new strategies for the treatment of this disease. ${ }^{12}$

In the context of development of these novel therapies, it is therefore critical to understand the current use of prophylactic therapies in real-world clinical practice and evaluate how these drugs are used over time. This can be achieved by using health-related administrative databases as tools for tracking and monitoring drug use. The aims of this drug utilisation study were to (1) describe the utilisation patterns of migraine drugs, (2) evaluate adherence to prophylactic medications and (3) investigate drugrelated costs.

\section{METHODS}

\section{Study design}

A retrospective population-based study was performed using administrative health-related database in the primary care setting of Campania region, one of the largest Italian regions situated in the South of the country and representing approximately $10 \%$ of the Italian population (ie, 5.9 million inhabitants). We used the
Strengthening the Reporting of Observational Studies in Epidemiology cross-sectional checklist when writing our report. ${ }^{13}$

\section{Data sources}

The data required for this study were retrieved from the Campania Regional Database for Medication Consumption and the discharge record database held by the regional hospital. The first database contains the records of drugs dispensed by community pharmacies and reimbursed by Local Health Authorities. Further details relating to data sources have been published previously. ${ }^{14}$ Data were collected between 1 January 2016 and 31 December 2018. All data sources were matched by record-linkage analysis via the use of a unique encrypted personal identification code and linked to the civil registry in order to collect important demographic information, including age, gender and date of death or migration. The WHO's International Classification of Diseases, Ninth Revision (ICD-9) was used to classify cases of migraine, and the Anatomical Therapeutic Chemical (ATC) code to classify active substances.

\section{Study population}

The study population included all subjects who were alive and residing in the Campania region during the study period. Figure 1 shows a flowchart depicting how patients were selected.

The presence of migraine was defined by any diagnosis of migraine as primary or secondary hospital discharge or at least two medical dispensations of migraine-specific acute or prophylactic medication (triptans, ATC IV:

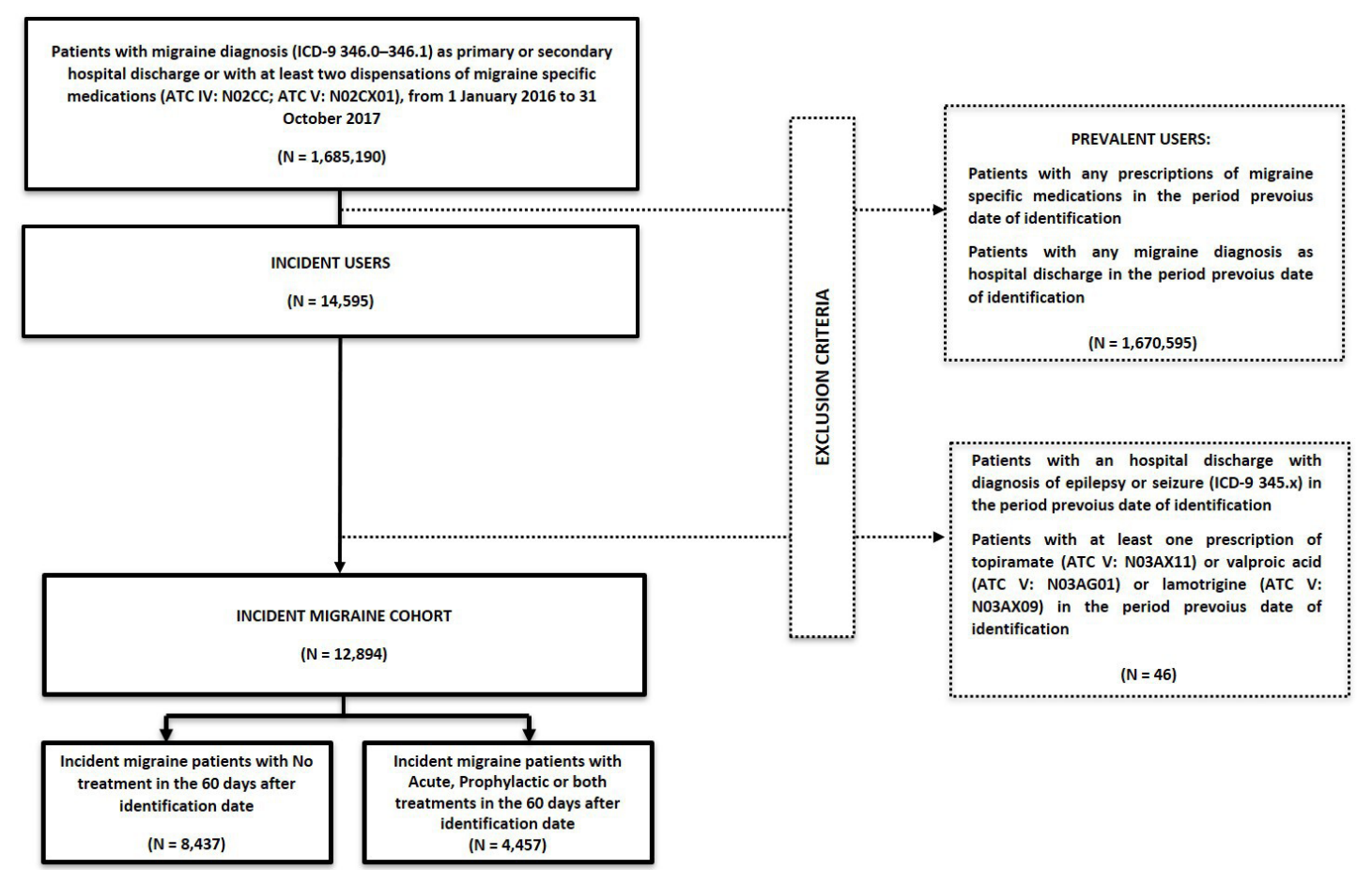

Figure 1 Study flow chart. ATC, Anatomical Therapeutic Chemical; ICD-9, International Classification of Diseases, Ninth Revision. 
N02CC or pizotifen, ATC V: N02C×01) between 1 January 2016 and 31 October 2017 (the recruitment period). The date of identification (entry into the migraine cohort) was defined as either the date of hospital discharge or the second drug dispensation, depending on which occurred first.

In order to identify incident users, it was applied a washout period prior to the date of identification in order to exclude all individuals with a previous diagnosis of migraine or those with a relevant history of medication. Furthermore, patients with a diagnosis of epilepsy or seizure (ICD-9 345.X) or with a dispensation of topiramate (ATC V: N03A×11), valproic acid (ATC V: N03AG01) or lamotrigine (ATC V: N03A $\times 09$ ), between 1 January 2012 until the date of entry into the migraine cohort, were also excluded in order to avoid misclassification. This procedure was followed because these drugs are specifically indicated for epilepsy and seizure.

Following the protocol adopted by Thomsen et al, ${ }^{16}$ patients were followed-up for 60 days after the day of identification. Within this timeframe, four incident treatment cohorts were identified based on the initial migraine treatment: (1) 'no treatment', defined as patients who received no migraine treatment; (2) 'acute treatment', defined as patients who received at least one specific or non-specific acute treatment for migraine; (3) 'prophylactic treatment', defined as patients who received at least one specific or non-specific prophylactic treatment for migraine and (4) 'both acute and prophylactic treatment', defined as patients who received both acute and prophylactic treatments for migraine that were either specific or non-specific. The drugs encountered in this study are listed in online supplemental table 1 . After this initial timeframe, patients were followed-up until the end of the study period ( 365 days). Figure 2 shows a schematic representation of the study.

\section{Adherence to therapy}

The main outcome of the present study was the adherence of patients to prophylactic migraine therapy. Adherence to therapy was evaluated in accordance with the European Society for Patient Adherence, Compliance and Persistence Medication Adherence Reporting Guideline, which recommends standard reporting approaches that are based on accepted taxonomy. This guideline divides adherence to medication into three interrelated yet distinct phases: initiation, implementation and persistence. ${ }^{17}$ The present study is focused on the persistence phase.

One year after the initiation of therapy, the persistence of all patients receiving prophylactic medications was measured. Persistence was defined as the continuation of treatment for 1 year after the index date and was estimated by measuring the time gap between a drug dispensation and the following one. The number of days of medication supplied in each package was calculated from the number of defined daily doses (DDDs) contained in the package. Persistence was identified as refilling a prescription for a prophylactic drug during the time period corresponding to all DDDs in the package prescribed previously, plus the following 60 days (the grace period). Subjects were considered to be non-persistent if the gap between two refills exceeded the grace period. Sensitivity analyses were performed with a 30-day and 90-day gap. Persistence to medication was measured at the drug class level (ATC V level).

Non-persistent subjects were categorised as: restarting (reinitiation of the same pharmacological treatment received at the index date); switching (reinitiation of a pharmacological treatment that was different from that dispensed at the index date) and full discontinuers (definitive interruption of a prophylactic migraine treatment).

\section{Covariates}

The following variables were assessed at baseline: sex, age, type of patient recruitment, comorbidities, comedications and the number of concomitant drugs (polypharmacy). Polypharmacy was defined according to three classes: 'excessive polypharmacy' was defined as the use of ten or more drugs; 'polypharmacy' as the use of five to nine drugs and 'no-polypharmacy' as the concomitant use of four drugs or fewer.

The time to treatment switching, restarting or full discontinuation was calculated as the median number of days and IQR. Comorbidities and comedications are summarised in online supplemental tables 2 and 3, respectively.

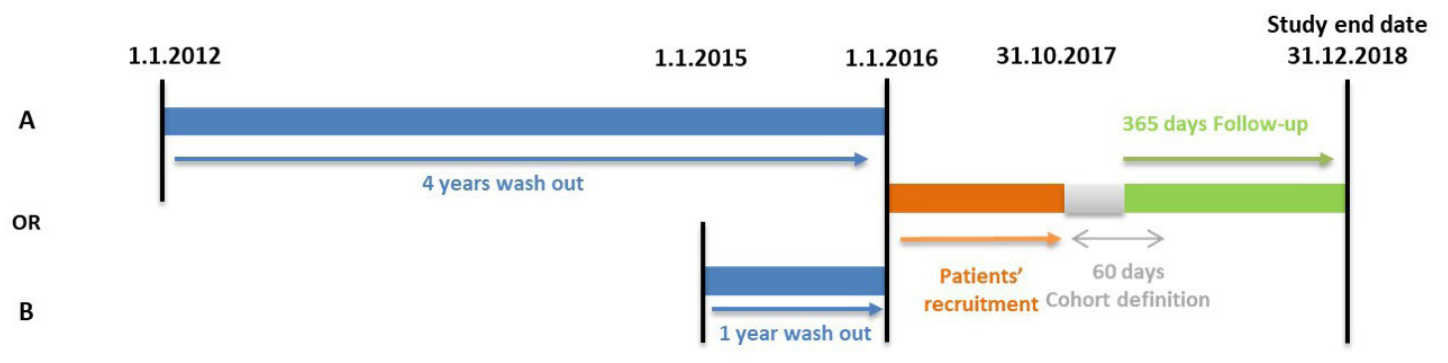

A. Hospital discharge

B. Pharmaceutical records

Figure 2 Schematic presentation of study design and patients' selection. 


\section{Statistical analyses}

Descriptive analysis was performed for all patient characteristics and the initial patterns of prophylactic migraine treatment. Differences between patient characteristics were compared using the chi-squared test for categorical variables or the unpaired t-test for numerical variables, as appropriate. A $p$ value $<0.05$ was considered to indicate statistical significance. Persistence rates were estimated using the Kaplan-Meier method and statistical differences were assessed between curves using the log-rank test.

Data management was performed with Microsoft SQL server (V.2018), and all analyses were performed with SPSS software for Windows (V.17.1, SPSS) and platform R (V.3.6, The R Formulation for Statistical Computing, Vienna, Austria).

\section{Cost analysis}

Finally, the drug costs of specific and non-specific migraine treatments were evaluated. Costs were expressed in Euro $(€)$ currency and are presented as the annual median cost per patient. Cost analysis was conducted from the perspective of the third-party payer, the National Health Service (NHS). In Italy, the NHS is responsible for financing and providing healthcare services. The total treatment cost was computed by multiplying the number of drugs prescribed during the entire follow-up period by the unit cost of the drug. This was made for each treatment cohort: acute, prophylactic, both acute and prophylactic. Drugs were costed according to the purchase price incurred by the NHS. The cost of each drug was calculated with reference to the time at which it was dispensed.

\section{Patient and public involvement}

No patients involved.

\section{RESULTS}

\section{Study population characteristics}

Our analyses identified 1685190 patients who were prescribed at least one drug for the treatment of migraine between 1 January 2016 and 31 December 2017. Of these, 14595 were incident patients with migraine; after applying the exclusion criteria, the final cohort of incident patients with migraine included 12894 subjects (figure 1). Characterisation of the incident migraine cohort over the first 60 days after the date of identification revealed 8437 patients who did not receive treatment. Consequently, the remaining 4457 patients received treatment and were included in the final analysis cohort. The characteristics of the study cohort at baseline are shown in table 1. Most patients $(81.1 \%)$ received acute treatment only as their index migraine treatment regimen, $10.7 \%$ of patients received prophylactic treatment and $8.2 \%$ of patient received both acute and prophylactic treatment. Median patient age was 49.8 years, and approximately two-thirds of the study cohort were women. A higher proportion of patients $(88.4 \%)$ were recruited via the prescription of migraine-specific treatments; only a minor percentage
(11.6\%) were recruited via hospital discharge records with a diagnosis of migraine. The most common comedications were cardiovascular drugs $(35.7 \%)$; the next most common drugs were those taken for the respiratory system $(16.1 \%)$. It was observed that $50.7 \%$ of the total incident migraine cohort were prescribed up to 4 comedications, $29.8 \%$ of patients were prescribed with 5-9 comedications and $19.5 \%$ of patients were prescribed with over 10 comedications.

Table 2 lists the types of initial specific or non-specific treatments that were taken acutely or prophylactically, stratified by sex. Of the subjects receiving only acute treatment, most (53\%) received triptan; the next most common treatment in this cohort was non-steroidal antiinflammatory drugs (NSAIDs) (31.4\%). Approximately $13 \%$ of patients received more than one specific or nonspecific acute medication simultaneously. Of the subjects receiving only prophylactic treatment, $31.7 \%$ received anticonvulsants (such as valproic acid or lamotrigine), $20 \%$ received a specific treatment (such as pizotifen), while $18.7 \%$ received antidepressants (such as amitriptyline or mirtazapine).

\section{Adherence to therapy}

Analysis of persistence in the cohort of patients receiving only prophylactic treatment was analysed 1 year after the initiation of therapy (table 3); this analysis involved 599 patients. During the 1 year follow-up period, $26.2 \%$ of these patients were persistent to their initial prophylactic treatment, while $73.8 \%$ had discontinued their initial treatment. Among the latter patients, $46.4 \%$ had fully discontinued the treatment within 103 days (IQR: 89.0), $31 \%$ had restarted treatment with the same prophylactic medication 46 days after the interruption (IQR 60.0) and $22.6 \%$ of patients had switched to another prophylactic medication within 98 days (IQR 57.5) (table 3). Figure 3 shows the results obtained from Kaplan-Meier analysis. Compared with men, women were significantly less likely to be persistent to prophylactic treatment (log-rank, $\mathrm{p}<0.001)$. In addition, Kaplan-Meier analysis was used to identify the proportion of persistent patients, as grouped by the class of prophylactic medication taken. At 365 days, the number of patients who remained on specific treatments were as follows: anticonvulsants $(38.3 \%)$, beta-blockers $(31.4 \%)$, antiepileptics $(22.7 \%)$, antidepressants $(21.8 \%)$ and antiserotonin agents $(13.8 \%)$.

\section{Cost analysis}

Figure 4 shows the median annual pharmaceutical costs stratified by the treatment typology of incident patients with migraine. The annual median drug cost per patient treated with acute medications was $€ 103$; the cost per patient for those taking prophylactic medications was $€ 75$. The annual cost per patient for those taking both acute and prophylactic treatments was $€ 163$. 
Table 1 Characteristics of the sample at time of index date

\begin{tabular}{|c|c|c|c|c|c|}
\hline & $\begin{array}{l}\text { Total, } \\
\mathrm{N}=4457 \\
(100 \%)\end{array}$ & $\begin{array}{l}\text { Acute } \\
\text { treatment, * } \\
\mathrm{N}=3613 \\
(81.1 \%)\end{array}$ & $\begin{array}{l}\text { Prophylactic } \\
\text { treatment, } \\
\mathrm{N}=477 \\
(10.7 \%)\end{array}$ & $\begin{array}{l}\text { Both,* } \\
\text { N=367 } \\
(8.2 \%)\end{array}$ & $P$ value \\
\hline \multicolumn{6}{|l|}{ Sex } \\
\hline Males & 1393 (31.3) & $1121(31.0)$ & $159(33.3)$ & $113(30.8)$ & \\
\hline Females & $3064(68.7)$ & 2492 (69.0) & $318(66.7)$ & $254(69.2)$ & \\
\hline Age groups & & & & & $<0.001$ \\
\hline$\leq 29$ years & $509(11.4)$ & $366(10.1)$ & $92(19.3)$ & $51(13.9)$ & \\
\hline 30-55 years & $2413(54.1)$ & $2000(55.4)$ & $195(40.9)$ & $218(59.4)$ & \\
\hline$\geq 56$ years & 1535 (34.4) & 1247 (34.5) & $190(39.8)$ & $98(26.7)$ & \\
\hline Comorbidities & & & & & $<0.001$ \\
\hline Autoimmune disease & $52(1.2)$ & $37(1.0)$ & $6(1.3)$ & $9(2.5)$ & \\
\hline Chronic kidney disease & $48(1.1)$ & $35(1.0)$ & $10(2.1)$ & $3(0.8)$ & \\
\hline COPD & 797 (17.9) & $651(18.0)$ & $79(16.6)$ & 67 (18.3) & \\
\hline Diabetes & $361(8.1)$ & $288(8.0)$ & $49(10.3)$ & $24(6.5)$ & \\
\hline Hypertension & 1485 (33.3) & 1178 (32.6) & $187(39.2)$ & $120(32.7)$ & \\
\hline Comedications & & & & & $<0.001$ \\
\hline Drugs for respiratory system & $717(16.1)$ & $596(16.5)$ & 63 (13.2) & $58(15.8)$ & \\
\hline Anticonvulsants & $323(7.2)$ & $243(6.7)$ & $51(10.7)$ & $29(7.9)$ & \\
\hline
\end{tabular}

*Acute cohort includes incident patients who have received a prescription of acute medication for migraine treatment 60 days after their index date. Prophylactic cohort includes incident patients who have received a prescription of prophylactic medication for migraine treatment 60 days after their index date. Both includes incident patients who have received a prescription of both acute and prophylactic medications for migraine treatment 60 days after their index date.

COPD, chronic obstructive pulmonary disease.

\section{DISCUSSION}

In this retrospective population-based study, the clinical characteristics and initial treatment pattern of incident patients with migraine living in the Campania region of Southern Italy were analysed. Our analysis showed that fewer than $20 \%$ of newly treated subjects began prophylactic migraine therapy. Furthermore, remarkably, $73.8 \%$ of these subjects discontinued their initial prophylactic treatment after approximately $3 \frac{1}{2}$ months; it was founded that only half of these patients resumed therapy (either by switching to another drug or restarting the same medication).

To the best of our knowledge, this is the first Italian study to investigate the patterns of persistence in incident patients with migraine treated with prophylactic medications. The present study also involved a database of drug prescriptions within a stable and specific geographic population. By characterising the use of migraine treatment in this context, it is possible to obtain useful data regarding the dynamics of therapy in the realworld setting.

The subjects analysed in this study mainly received acute medications; this is in line with other studies. Indeed, this pattern of usage has been described in publications from many different countries, including Denmark, Japan and the USA. ${ }^{16-20}$ A recent retrospective study stated that prophylactic medications are used less frequently than acute medications; furthermore and in line with our present results, patients treated with prophylactic medications showed a high rate of discontinuation following a 
Table 2 Type of migraine treatment dispensed within 60 days following the entry in the incident migraine cohort

\begin{tabular}{|c|c|c|c|c|}
\hline \multirow[b]{3}{*}{ Migraine treatment } & \multicolumn{4}{|c|}{ Incident migraine cohort } \\
\hline & Total & Males & Females & \multirow[b]{2}{*}{ P value } \\
\hline & $\begin{array}{l}N=4457 \\
(\%)\end{array}$ & $\mathrm{N}=1393(\%)$ & $\mathrm{N}=3064(\%)$ & \\
\hline Treatment typology & & & & $<0.001$ \\
\hline Acute* & $3613(81.1)$ & $1121(80.5)$ & $2492(81.3)$ & \\
\hline \multicolumn{5}{|l|}{ Specific acute treatment† } \\
\hline $\begin{array}{l}\text { Triptans (sumatriptan, zolmitriptan, rizatriptan, almotriptan, } \\
\text { eletriptan, frovatriptan) }\end{array}$ & $1915(53.0)$ & $567(50.6)$ & $1348(54.1)$ & \\
\hline \multicolumn{5}{|l|}{ Non-specific acute treatment $\dagger$} \\
\hline Antiemetics (ondansetron) & $9(0.2)$ & $5(0.4)$ & $4(0.2)$ & \\
\hline Aspirin & $5(0.1)$ & $1(0.1)$ & $4(0.2)$ & \\
\hline Drugs for functional gastrointestinal disorders (metoclopramide) & $12(0.3)$ & $4(0.4)$ & $8(0.3)$ & \\
\hline $\begin{array}{l}\text { NSAIDs (diclofenac, flurbiprofen, ibuprofen, indometacin, } \\
\text { ketoprofen, ketorolac, naproxen, nimesulide, piroxicam) }\end{array}$ & $1136(31.4)$ & $380(33.9)$ & $756(30.3)$ & \\
\hline Opioids (tramadol, codeine/paracetamol) & $61(1.7)$ & $17(1.5)$ & $44(1.8)$ & \\
\hline More than one specific/non-specific acute medication $\dagger$ & $475(13.1)$ & $147(13.1)$ & $328(13.2)$ & \\
\hline Prophylactic* & $477(10.7)$ & $159(11.4)$ & $318(10.4)$ & \\
\hline \multicolumn{5}{|l|}{ Specific prophylactic treatmentł } \\
\hline Antiserotonin agents (pizotifen) & $95(19.9)$ & $28(17.6)$ & $67(21.1)$ & \\
\hline \multicolumn{5}{|l|}{ Non-specific prophylactic treatmentł } \\
\hline Antidepressants (amitriptyline, mirtazapine) & $89(18.7)$ & $17(10.7)$ & $72(22.6)$ & \\
\hline Beta-blockers (metoprolol, propranolol) & $40(8.4)$ & $19(11.9)$ & $21(6.6)$ & \\
\hline Other anticonvulsants (valproic acid, lamotrigine) & $151(31.7)$ & $76(47.8)$ & $75(23.6)$ & \\
\hline Antiepileptics (topiramate) & $83(17.4)$ & $16(10.1)$ & $67(21.1)$ & \\
\hline More than one specific/non-specific prophylactic medicationł & $19(4.0)$ & $3(1.9)$ & $16(5.0)$ & \\
\hline Both acute and prophylactic medication* & $367(8.2)$ & $113(8.1)$ & $254(8.3)$ & \\
\hline
\end{tabular}

*The percentage was calculated on the total of treated patients.

†The percentage was calculated on the total of patients received acute treatment.

$\ddagger$ The percentage was calculated on the total of patients received prophylactic treatment.

NSAIDs, Non-Steroidal Anti-inflammatory Drugs.

brief treatment period. ${ }^{18}$ The authors of this earlier study also reported rates of re-initiation and switching that were comparable to our present results. ${ }^{18}$

A recent Italian drug utilisation study suggested that there was an unmet need in the management of migraine and that only $9.9 \%$ of patients with migraine are treated with prophylactic drugs. ${ }^{21}$

In the USA, Woolley et $a l^{22}$ reported low rates of persistence to migraine prophylactics, thus confirming the trend for early therapeutic discontinuation in these patients. Woolley $e t a l^{22}$ further observed that opioids were the most commonly prescribed non-specific acute medication. In this regard, our current data are slightly different: the most common treatment regimen prescribed was NSAIDs (31.4\%) and rarely, opioids (1.7\%). This difference can be explained by the fact that the use of opioids for pain treatment is common in the USA; in contrast, NSAIDs are preferred in Italy. However, it is evident that trends are beginning to change in the US. This follows a
Food and Drug Administration warning in March 2016 relating to the co-prescription of opioid, selective serotonin reuptake inhibitors and serotonin norepinephrine reuptake inhibitors or opioid-triptan and the risk of serotonin syndrome. ${ }^{23}$ It is also interesting to note that our analysis showed a remarkable proportion of switchers among patients using pizotifen $(42.5 \%)$. This can be related to the fact that this drug ceased to be available on the Italian market in February 2018. ${ }^{24}$

In addition, the cost aspect of migraine treatment is not negligible. Our results showed that the median annual cost per patient taking both acute and prophylactic migraine treatment was $€ 163$, this is slightly lower than other evidences, such as the case of Latvia recording $€ 801$ annually per patient, and $€ 721$ in Lithuania, and in these latters countries two-thirds of total cost were related to lost workdays due to absenteeism and presenteeism. ${ }^{25}$ Therefore, improving and implementing the care of patients with migraine, such as through a wider 
Table 3 One-year persistence with prophylactic migraine treatment

\begin{tabular}{|c|c|c|c|c|c|c|c|}
\hline & \multirow{2}{*}{$\begin{array}{l}\text { Total } \\
N=599^{\star} \\
(100 \%)\end{array}$} & \multirow{2}{*}{$\begin{array}{l}\begin{array}{l}\text { Antiserotonin } \\
\text { agents }\end{array} \\
\mathrm{N}=123(20.5 \%)\end{array}$} & \multirow{2}{*}{$\begin{array}{l}\text { Antidepressants } \\
\mathrm{N}=110(18.4 \%)\end{array}$} & \multirow{2}{*}{$\begin{array}{l}\text { Beta-blockers } \\
\mathrm{N}=51(8.5 \%)\end{array}$} & \multirow{2}{*}{$\begin{array}{l}\text { Antiepileptics } \\
\mathrm{N}=132(22.0 \%)\end{array}$} & \multirow{2}{*}{$\begin{array}{l}\begin{array}{l}\text { Other } \\
\text { anticonvulsants }\end{array} \\
\mathrm{N}=183(30.6 \%)\end{array}$} & \multirow[b]{2}{*}{$P$ value } \\
\hline & & & & & & & \\
\hline Persistent patients $\dagger$ & $157(26.2)$ & $17(13.8)$ & $24(21.8)$ & $16(31.4)$ & $30(22.7)$ & $70(38.3)$ & $<0.001$ \\
\hline Non-peristent patients $†$ & $442(73.8)$ & $106(86.2)$ & $86(78.2)$ & $35(68.6)$ & $102(77.3)$ & $113(61.7)$ & $<0.001$ \\
\hline Full discontinuer $\neq$ & $205(46.4)$ & $42(39.6)$ & $43(50.0)$ & $12(34.3)$ & $46(45.1)$ & $62(54.9)$ & \\
\hline Switch $\ddagger$ & $100(22.6)$ & $45(42.5)$ & $19(22.1)$ & $5(14.3)$ & $20(19.6)$ & $11(9.7)$ & \\
\hline $\begin{array}{l}\text { Days on treatment before } \\
\text { stopping (discontinuation), } \\
\text { median (IQR) }\end{array}$ & $103.0(89.0)$ & $110.0(79.0)$ & $97.0(120.0)$ & $93.0(78.5)$ & $90.5(71.0)$ & $108.5(87.0)$ & \\
\hline $\begin{array}{l}\text { Days after treatment } \\
\text { interruption before } \\
\text { restarting, median (IQR) }\end{array}$ & $46.0(60.0)$ & $39.0(109.0)$ & $59.5(83.0)$ & $65.0(64.0)$ & 26.5 (32.0) & $50.0(66.0)$ & \\
\hline
\end{tabular}

*Number of patients with prophylactic treatment is higher than that reported in table 2 as some patients who started treatment with both acute and prophylactic medications, later continued their treatment only with prophylactic medication.

$\dagger$ The percentage was calculated on the total of treated patients.

$\ddagger$ The percentage was calculated on the total of non-persistent patients.

availability of prophylactic drugs, would lead to higher direct costs, but this cost increase could be balanced by a lower loss of productivity related to migraine.

This study was limited by the very nature of the Italian administrative databases used to obtain data. For example, our data source does not track diagnostic information. Therefore, patient identification had to be performed ex post by using proxy drugs that are reimbursed by the NHS. To mitigate the impact of this limitation the authors adopted the same methodology as that used previously by Thomsen $e$ al. ${ }^{16}$ It is also possible that some patients with migraine may not have been identified because they used drugs that are not reimbursable by the NHS (eg, ergots ATC IV: N02CA; flunarizine ATC V: N07CA03). For this reason, the no-treatment cohort $(n=8437)$ may have included some subjects that were actually taking these drugs. Therefore, in this study, there could be an underestimation of the prevalence and incidence of migraine. Another limitation, also related to the administrative database, was the lack of information relating to the specific reasons for treatment discontinuation.

In general, the results of this study highlight the unmet need for an effective and sustainable therapy for migraine. Indeed, current prophylactic therapies can suffer from a lack of specificity, poor tolerability, potential side effects and limited efficacy. Collectively, these factors lead to dissatisfaction in a large proportion of patients, thus resulting in low adherence to treatment. ${ }^{26}$ Furthermore, non-adherence to medication could reduce the efficacy of pharmacological therapies; this may simultaneously increase the direct and indirect costs related to such treatment. ${ }^{27-32}$ These factors are driving research and significant advances in the prophylactic treatment of migraine, including the generation of anti-CGRP
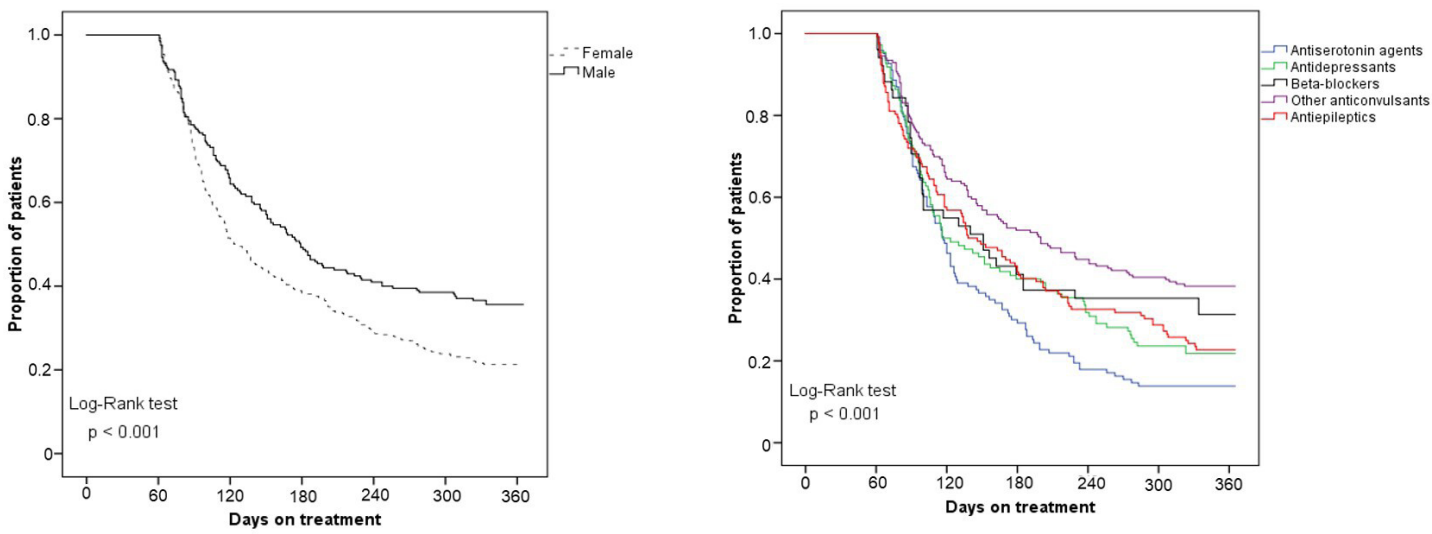

Figure 3 Time to discontinuation up to 365 days' follow-up from the initial prophylactic treatments, stratified by sex and drug category at index date. 


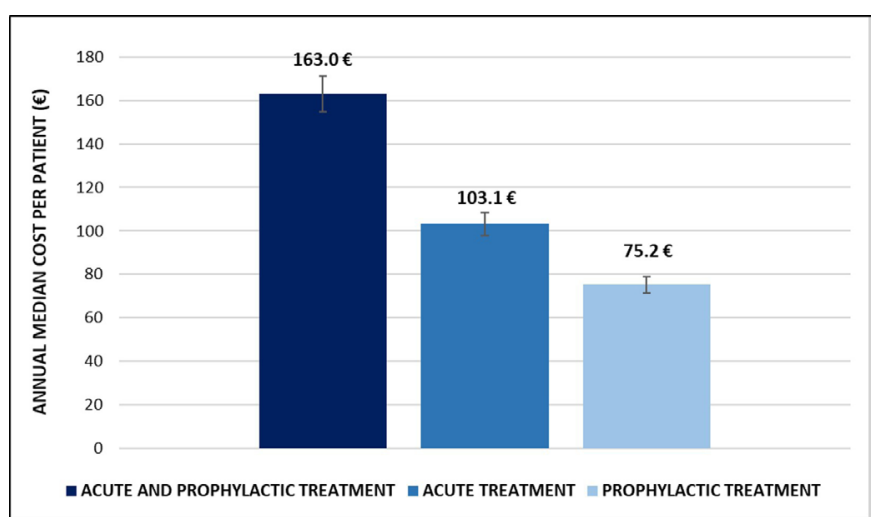

Figure 4 Annual median cost per patient $(€)$ stratified by treatment typology.

monoclonal antibodies. These drugs are useful for migraine-specific prophylactic treatment and appear to perform better than current therapies. ${ }^{33}{ }^{34}$ However, the high costs related to these emerging drugs could represent a significant issue when selecting the patients with migraine who would benefit. In this promising scenario, the provision of information based on real-world data represents a highly useful way to support stakeholders towards creating better management strategies for this disease. ${ }^{35-38}$

\section{CONCLUSION}

The present analysis showed that the treatment of migraine with acute medications is still prevalent in the Italian clinical practice. Only a small number of patients began prophylactic treatment and persistence was poor; these observations were not consistent with the clinical guidelines for primary headaches. In addition, most of the non-persistent patients tended to quit prophylactic therapy within 3 months of starting treatment. These findings reflect an unmet need for improved prophylactic therapies in order to improve the management strategies used for migraine. This unmet need must be addressed in future treatment guidelines. New monoclonal antibodies for the specific prophylactic treatment of migraine could represent a significant opportunity for improving long-term persistence and ensuring therapeutic efficacy in order to reduce the number of acute migraine episodes. Analysis of patient characteristics could help to identify patients who would specifically benefit from these emerging therapies or design a more effective clinical management plan for existing prophylactic migraine medications. These scenarios may require further research to be based on real-world data in order to enable investigation of the dynamics underlying the prophylactic treatment of migraine and to optimise resources, so that disease management can be improved.

Correction notice This article has been corrected since it was first published. The funding statement has been updated.

Contributors V0, SM and EM conceived and designed the study. SM analysed the data and wrote the manuscript. V0, UT and EM critically reviewed the manuscript for important intellectual content and contributed to the discussion. VMM extracted and analyses data. VO and SM interpreted the data. EM provided study supervision. All authors gave their final approval for the final version of the manuscript to be published and agree to be accountable for all aspects of the work.

Funding This work was supported by the Regional Project of Italian Medicine Agency (AIFA) "Analisi delle Prescrizioni Farmaceutiche in Regione Campania" (Pharmacovigilance grant).

Competing interests None declared.

Patient consent for publication Not required.

Ethics approval All procedures performed, in this study, were in accordance with the current national law from the Italian Medicines Agency. The article does not contain clinical studies, and all patients' data were fully anonymised. For this type of study, formal consent was not required. Permission to use anonymised data for the present study was granted by the responsible authority, 'Unità del Farmaco, Regione Campania'.

Provenance and peer review Not commissioned; externally peer reviewed.

Data availability statement No data are available. The datasets for this manuscript are not publicly available because the data set was only accessed and analysed by the authors who are affiliates to CIRFF, University of Naples Federico II. Access to the data is allowed only to affiliates due to Campania region policies.

Supplemental material This content has been supplied by the author(s). It has not been vetted by BMJ Publishing Group Limited (BMJ) and may not have been peer-reviewed. Any opinions or recommendations discussed are solely those of the author(s) and are not endorsed by BMJ. BMJ disclaims all liability and responsibility arising from any reliance placed on the content. Where the content includes any translated material, BMJ does not warrant the accuracy and reliability of the translations (including but not limited to local regulations, clinical guidelines, terminology, drug names and drug dosages), and is not responsible for any error and/or omissions arising from translation and adaptation or otherwise.

Open access This is an open access article distributed in accordance with the Creative Commons Attribution Non Commercial (CC BY-NC 4.0) license, which permits others to distribute, remix, adapt, build upon this work non-commercially, and license their derivative works on different terms, provided the original work is properly cited, appropriate credit is given, any changes made indicated, and the use is non-commercial. See: http://creativecommons.org/licenses/by-nc/4.0/.

ORCID iD

Sara Mucherino http://orcid.org/0000-0003-3357-5655

\section{REFERENCES}

1 Victor TW, Hu X, Campbell JC, et al. Migraine prevalence by age and sex in the United States: a life-span study. Cephalalgia 2010;30:1065-72.

2 Patel NV, Bigal ME, Kolodner KB, et al. Prevalence and impact of migraine and probable migraine in a health plan. Neurology 2004;63:1432-8.

3 Lipton RB, Bigal ME, Diamond M, et al. Migraine prevalence, disease burden, and the need for preventive therapy. Neurology 2007;68:343-9.

4 Stovner LJ, Andrée C, Eurolight Steering Committee. Impact of headache in Europe: a review for the Eurolight project. $J$ Headache Pain 2008;9:139-46.

5 Bloudek LM, Stokes M, Buse DC, et al. Cost of healthcare for patients with migraine in five European countries: results from the International burden of migraine study (IBMS). J Headache Pain 2012;13:361-78.

6 Stovner L, Hagen K, Jensen R, et al. The global burden of headache: a documentation of headache prevalence and disability worldwide. Cephalalgia 2007;27:193-210.

7 Sarchielli P, Granella F, Prudenzano MP, et al. Italian guidelines for primary headaches: 2012 revised version. J Headache Pain 2012;13 Suppl 2:31-70.

8 Parikh SK, Silberstein SD. Preventive treatment for episodic migraine. Neurol Clin 2019;37:753-70.

9 Berger A, Bloudek LM, Varon SF, et al. Adherence with migraine prophylaxis in clinical practice. Pain Pract 2012;12:541-9.

10 Ong JJY, Wei DY-T, Goadsby PJ. Recent advances in pharmacotherapy for migraine prevention: from pathophysiology to new drugs. Drugs 2018;78:411-37. 
11 Barbanti P, Ferroni P. Onabotulinum toxin A in the treatment of chronic migraine: patient selection and special considerations. J Pain Res 2017;10:2319-29.

12 Yuan $\mathrm{H}$, Lauritsen CG, Kaiser EA, et al. CGRP monoclonal antibodies for migraine: rationale and progress. BioDrugs 2017;31:487-501.

13 von Elm E, Altman DG, Egger M, et al. The strengthening the reporting of observational studies in epidemiology (STROBE) statement: guidelines for reporting observational studies. J Clin Epidemiol 2008;61:344-9.

14 Juste AM, Menditto E, Orlando V, et al. Treatment patterns of diabetes in Italy: a population-based study. Front Pharmacol 2019;10:1-11.

15 Iolascon G, Gimigliano F, Orlando V, et al. Osteoporosis drugs in realworld clinical practice: an analysis of persistence. Aging Clin Exp Res 2013;25 Suppl 1:137-41.

16 Thomsen RW, Szépligeti SK, Xue F, et al. Patterns of initial migraine treatment in Denmark: a population-based study. Pharmacoepidemiol Drug Saf 2019;28:322-9.

17 De Geest S, Zullig LL, Dunbar-Jacob J, et al. ESPACOMP medication adherence reporting guideline (EMERGE). Ann Intern Med 2018;169:30-5.

18 Meyers JL, Davis KL, Lenz RA, et al. Treatment patterns and characteristics of patients with migraine in Japan: a retrospective analysis of health insurance claims data. Cephalalgia 2019;39:1518-34.

19 Hepp Z, Dodick DW, Varon SF, et al. Persistence and switching patterns of oral migraine prophylactic medications among patients with chronic migraine: a retrospective claims analysis. Cephalalgia 2017;37:470-85.

20 Bonafede M, Wilson K, Xue F. Long-Term treatment patterns of prophylactic and acute migraine medications and incidence of opioid-related adverse events in patients with migraine. Cephalalgia 2019;39:1086-98.

21 Agostoni E, Barbanti P, Frediani F, et al. Real-World insights on the management of migraine patients: an Italian nationwide study. Curr Med Res Opin 2019;35:1545-54.

22 Woolley JM, Bonafede MM, Maiese BA, et al. Migraine prophylaxis and acute treatment patterns among commercially insured patients in the United States. Headache 2017;57:1399-408.

23 Molina KC, Fairman KA, Sclar DA. Concomitant use of opioid medications with triptans or serotonergic antidepressants in US office-based physician visits. Drug Healthc Patient Saf 2018;10:37-43.

24 Agenzia Italiana del Farmaco (AIFA). Farmaci carenti. Available: https://www.aifa.gov.it/web/guest/farmaci-carenti [Accessed 20 Jan 2020].
25 Lublóy Ágnes. Economic burden of migraine in Latvia and Lithuania: direct and indirect costs. BMC Public Health 2019;19:1242.

26 Bigal M, Rapoport A, Aurora S, et al. Satisfaction with current migraine therapy: experience from 3 centers in US and Sweden. Headache 2007;47:475-9.

27 Iolascon G, Gimigliano F, Moretti A, et al. Rates and reasons for lack of persistence with anti-osteoporotic drugs: analysis of the Campania region database. Clin Cases Miner Bone Metab 2016;13:127-30.

28 Menditto E, Guerriero F, Orlando V, et al. Self-Assessment of adherence to medication: a case study in Campania region community-dwelling population. J Aging Res 2015;2015:1-6.

29 Illario M, Vollenbroek-Hutten M, Molloy DW, et al. Active and healthy ageing and independent living. J Aging Res 2015;2015:1-3.

30 Illario M, Vollenbroek-Hutten MMR, Molloy DW, et al. Active and healthy ageing and independent living 2016. J Aging Res 2016;2016:1-3.

31 Menditto E, Orlando V, Coretti S, et al. Doctors commitment and long-term effectiveness for cost containment policies: lesson learned from biosimilar drugs. Clinicoecon Outcomes Res 2015;7:575-81.

32 Guerriero F, Orlando V, Monetti VM, et al. Biological therapy utilization, switching, and cost among patients with psoriasis: retrospective analysis of administrative databases in southern Italy. Clinicoecon Outcomes Res 2017;9:741-8.

33 Agostoni EC, Barbanti P, Calabresi P, et al. Current and emerging evidence-based treatment options in chronic migraine: a narrative review. J Headache Pain 2019;20:92.

34 Burch R, Rayhill M. New preventive treatments for migraine. BMJ 2018;361:k2507-3.

35 Ruggeri M, Manca A, Coretti S, et al. Investigating the generalizability of economic evaluations conducted in Italy: a critical review. Value Health 2015;18:709-20.

36 Coretti S, Romano F, Orlando V, et al. Economic evaluation of screening programs for hepatitis $\mathrm{C}$ virus infection: evidence from literature. Risk Manag Healthc Policy 2015;8:45-54.

37 Cammarota S, Bruzzese D, Catapano AL, et al. Lower incidence of macrovascular complications in patients on insulin Glargine versus those on basal human insulins: a population-based cohort study in Italy. Nutr Metab Cardiovasc Dis 2014;24:10-17.

38 Scala D, Menditto E, Caruso G, et al. Are you more concerned about or relieved by medicines? An explorative randomized study of the impact of telephone counseling by pharmacists on patients' beliefs regarding medicines and blood pressure control. Patient Educ Couns 2018;101:679-86. 